\title{
Epstein-Barr Virus Positive Follicular Lymphoma of Lymph Node
}

\author{
In Ho Choi', So-Young Jin', Dong-Won Kim', Yoon-Mi Jeen', Kyung-Ha Kim², Jong Ho Won² \\ Departments of 'Pathology and ${ }^{2}$ Internal Medicine, Soonchunhyang University Seoul Hospital, Soonchunhyang University College of Medicine, Seoul, Korea
}

\begin{abstract}
Epstein-Barr virus (EBV) infection has been associated with a number of lymphoid malignancies, including endemic Burkitt's lymphoma, some classical Hodgkin's lymphoma, diffuse large B-cell lymphoma, extranodal NK/T cell lymphoma, and angioimmunoblastic T-cell lymphoma. A 59-year-old woman underwent an excisional biopsy for a left axillary mass under suspicion of malignant lymphoma. A preoperative radiological study revealed multiple enlarged lymph nodes at the left axilla, mesentery, and the left external iliac chain with hypermetabolism on a positron emission tomography-computed tomography scan. She was histologically diagnosed with EBV-positive follicular lymphoma (FL, grade 3a) and received the R-CHOP (rituximab, cyclophosphamide, hydroxydaunorubicin, vincristine, prednisone) chemotherapy regimen. Herein, we report a rare case of EBV associated FL of lymph node with its review of literature.
\end{abstract}

Keywords: Follicular lymphoma; Herpesvirus 4, human; Epstein-Barr virus-associated lymphoma

\section{INTRODUCTION}

Epstein-Barr virus (EBV) is a member of the herpes virus family and is associated with a number of lymphoid and non-lymphoid malignancies, particularly the development of certain lymphomas including endemic Burkitt's lymphoma, some classical Hodgkin's lymphomas, diffuse large B-cell lymphoma (DLBCL), extranodal NK/T cell lymphoma, and angioimmunoblastic T-cell lymphoma [1]. However, rare cases of follicular lymphoma (FL) with EBV infection and a few mimicking cases has been reported [2-6]. Herein, we report a rare case of EBV associated FL of lymph node with its review of literature.

\section{CASE REPORT}

A 59-year-old woman, who had no medical history, was presented at our hospital due to an incidentally discovered left axillary mass. She had no other clinical symptoms. The initial complete blood count showed following results: white blood cells, $9.7 \mathrm{~K} / \mu \mathrm{L}$ (neutrophils, 80.6\%; lymphocytes, 11.3\%; monocytes, 6.1\%); hemo- globin, $11.9 \mathrm{~g} / \mathrm{dL}$ (normal range, 12 to $16 \mathrm{~g} / \mathrm{dL}$ ); and platelets, $361 \mathrm{~K} /$ $\mu \mathrm{L}$. Serum lactate dehydrogenase had slightly increased to $223 \mathrm{U} / \mathrm{L}$ (normal range, 106 to $211 \mathrm{U} / \mathrm{L}$ ), but other laboratory findings were unremarkable. A neck computed tomography scan revealed multiple well-defined, homogeneous enhancing, and enlarged lymph nodes in the left axilla (Fig. 1A). A subsequent positron emission tomography-computed tomography scan revealed multiple enlarged lymph nodes in the left axilla (levels I and II) with hypermetabolism (maximum standardized uptake value [SUV], 14.64; average SUV, 4.70). Other hypermetabolic lymphadenopathy was detected in the mesentery and left external iliac chain with no suspicious foci at other organs that were compatible with lymphoma. An excisional biopsy of the left axillary mass was performed, and it was histologically confirmed as FL grade 3a. She received the R-CHOP (rituximab, cyclophosphamide, hydroxydaunorubicin, vincristine, prednisone) chemotherapy and was followed for 9 months without any complications.

The lymph nodes microscopically consisted of small areas of reactive follicles on the low power view (Fig. 2A, left lower side). Other areas showed variously sized follicles with a preserved mantle
Correspondence to: In Ho Choi

Department of Pathology, Soonchunhyang University Seoul Hospital, 59 Daesagwan-ro, Yongsan-gu, Seoul 140-743, Korea

Tel: +82-2-709-9440, Fax: +82-2-709-9441, E-mail: s78170@schmc.ac.kr

Received: Feb. 5, 2015/ Accepted after revision: Apr. 22, 2015
(C) 2015 Soonchunhyang Medical Research Institute This is an Open Access article distributed under the terms of the Creative Commons Attribution Non-Commercial License (http://creativecommons.org/licenses/by-nc/3.0/). 

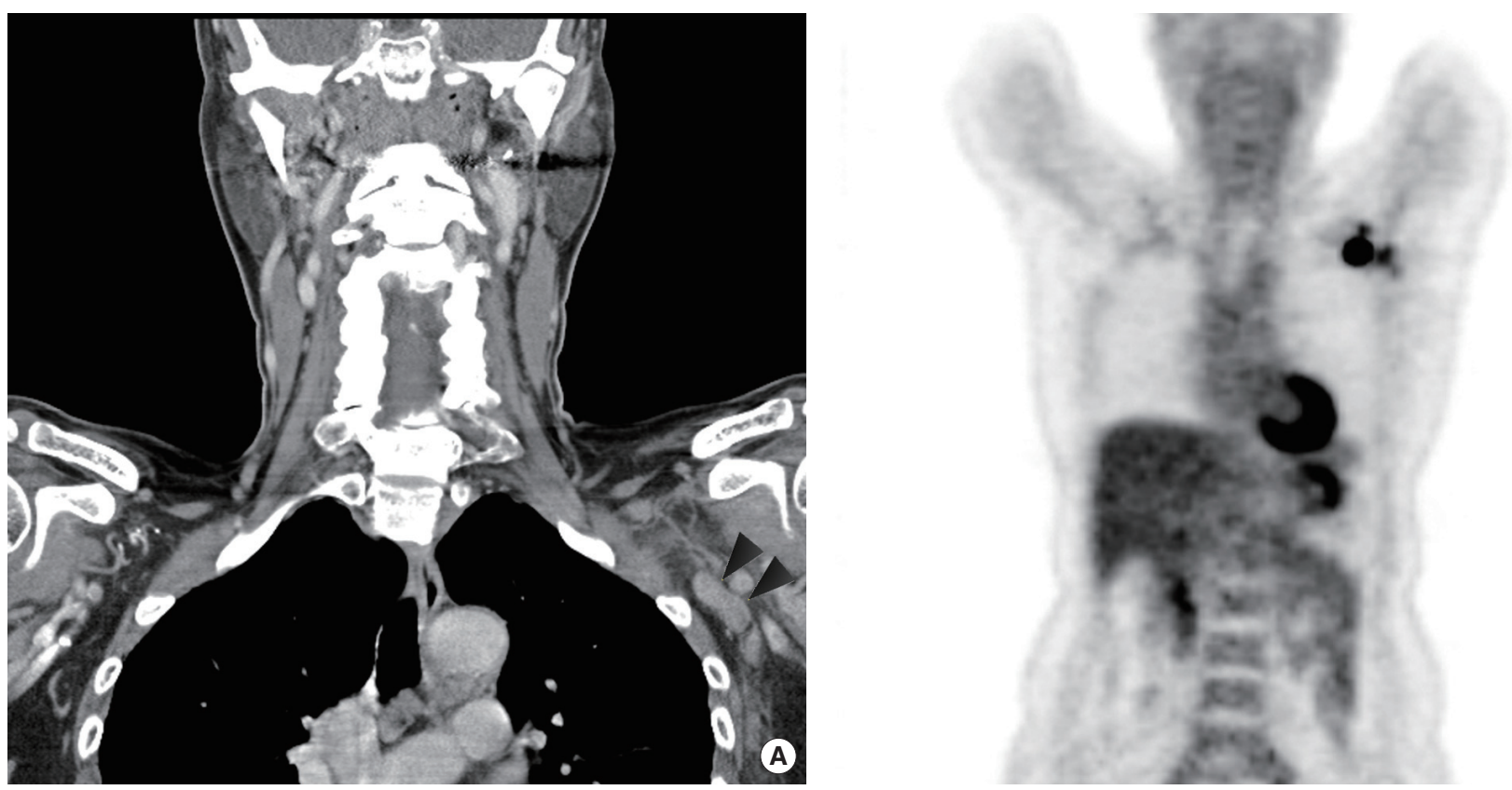

Fig. 1. Radiographic findings. (A) Computed tomography shows multiple, well-circumscribed, enlarged nodes in the left axilla (levels I and II) (arrowhead). (B) Positron emission tomography-computed tomography reveals hypermetabolic foci in the left axilla and mesentery.

zone (Fig. 2A, left upper side), and predominant areas containing vague follicles with peripheral fading and coalescence (Fig. 2A, right). Some follicles were separated by prominent lymphocytic mantle, and the neoplastic follicles showed a lack of cellular polarity (Fig. 2B). The neoplastic follicles contained mixed small cleaved cells without nucleoli (centrocytes) and larger non-cleaved cells with moderate cytoplasm, open chromatin, and multiple nucleoli (centroblasts) (Fig. 2C). More than 15 centroblasts/high power field (HPF) were present in the neoplastic germinal center (GC), consistent with grade $3 \mathrm{~A}$ FL. In addition, no diffuse area that fulfilled the criteria for diffuse large B cell lymphoma with FL was detected in any of the excised lymph nodes. Immunohistochemical staining showed tumor cells positive for CD20 and Bcl-6 and negative for CD3, CD5, CD10, Bcl-2, and cyclin D-1. The Ki-67 proliferation index increased in the neoplastic follicles with loss of polarity (Fig. 2D-H). An association with EBV was clearly demonstrated by the neoplastic follicles and tumor cells with EBV in situ hybridization (EBER) (Fig. 2I).

\section{DISCUSSION}

EBV is a member of herpes virus with overall seroprevalence $>90 \%$ in all adults. A spectrum of EBV-related diseases exists, such as a chronic active EBV infection and EBV-related epithelial, mesenchymal, or lymphoid malignancies [7].

Despite the growing prevalence of EBV-associated lymphomas including the heterogeneous group of B-cell lymphomas (Burkitt's lymphoma, DLBCL, primary effusion lymphoma, etc.) and NK/ T-cell lymphoma, the exact roles of EBV in lymphomagenesis remain unclear [1]. Moreover, since Seite et al. [6] suggested that EBV infection did not contribute significantly in Bcl-2 activation in the FL, an association between EBV infection and FL, and the optimal management of EBV-associated FL have been unsatisfactory. Although its incidence is extremely rare, there have been several studies including EBV-associated FL [4,5,8]. A study by Ishtiaq et al. [4] included only two EBV-positive cases out of 11 cases of FL. Sasikala et al. [9] reviewed the EBV-positive non-Hodgkin's lymphomas including six EBV-positive cases out of 17 cases with FL; the characteristics of EBV-positive FL included 48 years of median age (range, 34 to 70 years), 10:7 of sex ratio (male:female) and all type A strain [8]. However, EBV-associated FL has not yet been reported in Korea to our best knowledge.

Histologically, there can be several differential diagnoses in the present case, including EBV infected follicular hyperplasia and early phase of EBV-positive DLBCL or EBV-positive DLBCL with FL. Although Bcl-2 was negative in the neoplastic follicles, malignant transformation was histologically noted on the enlarged GCs of the present case, and these microscopic findings fulfilled the FL 

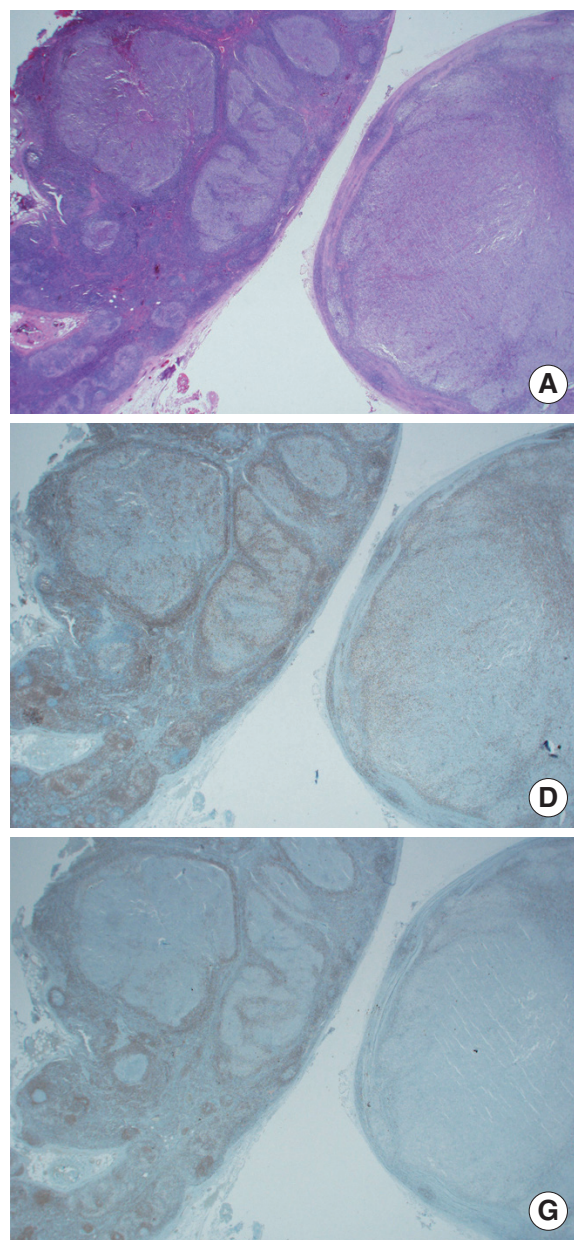

(G)
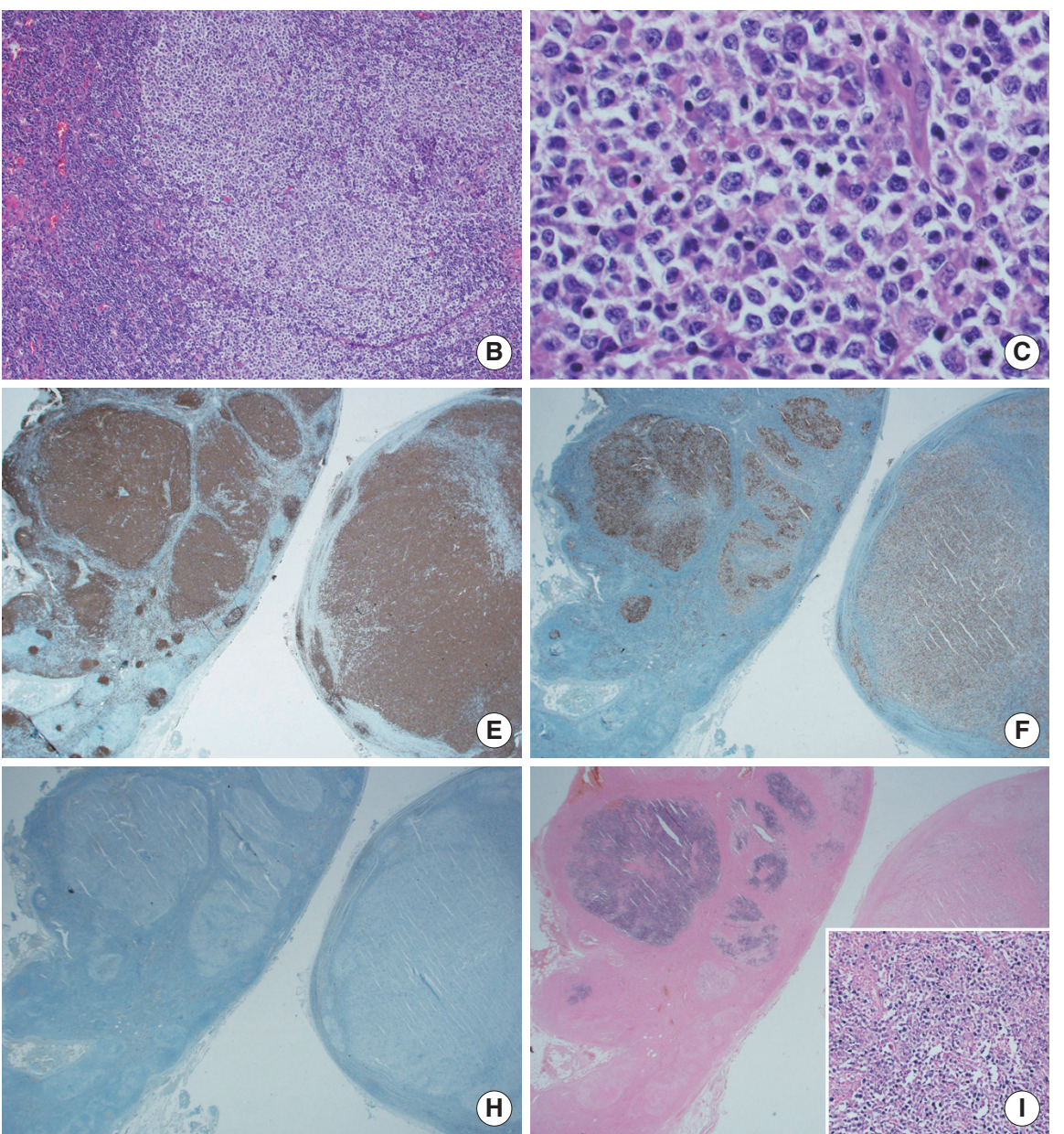

(H)

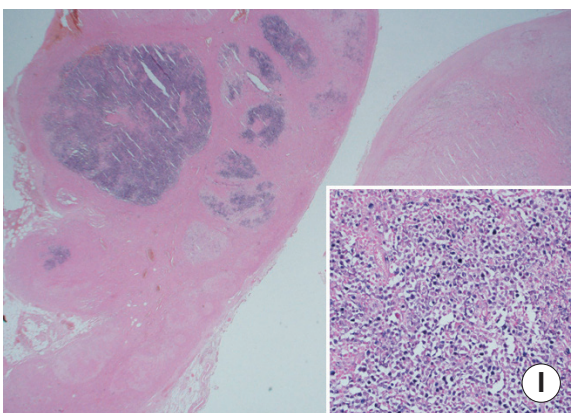

Fig. 2. Microscopic findings of Epstein-Barr virus (EBV)-positive follicular lymphoma. (A) One of the lymph nodes shows a reactive hyperplastic area (left lower side) and an area with lymphomatous change (left upper side). The other node shows vague follicles with peripheral fading and coalescence (right side) (H\&E, $\times 12.5)$. (B) Some neoplastic follicles are surrounded by a thick lymphocytic mantle with a lack of cellular polarization $(H \& E, \times 40)$. (C) The tumor cells consist of mixed centrocytes and centroblasts $(H \& E, \times 400)$. (D-H) The neoplastic follicles are negative for $C D 3(D)$ and positive for $C D 20(E)$, and the Ki-67 (F) proliferation index increased in the neoplastic follicles (left upper) with loss of polarity compared to those of reactive follicles (left lower). Tumor cells are for Bcl-2 (G) and CD10 negative (H). (I) The neoplastic follicles were positive on in situ hybridization for EBV. Inset: EBV-positive tumor cells $(\times 100)$.

grade 3A criteria; many neoplastic follicles with transformed GC contained atypical large lymphoid cells ( $>15$ centroblasts/HPF) with loss of polarity on Ki-67 staining. Although FL has been associated with Bcl-2 and CD10 positivity in GCs, several reports indicate that grade $3 \mathrm{FLs}$ can be negative for Bcl-2 (particularly in high grade $\mathrm{FL},<50 \%$ of $\mathrm{FL}$ grade 3$)$ and $\mathrm{CD} 10[9,10]$. According to Roughan et al. [11], EBV infected cells are known to express common GC markers (CD10 and Bcl-6) and overexpress Bcl-2. However, the immunohistochemical profile of EBV-infected neoplastic B cells is not well characterized. In the present case, EBV was detected only in the neoplastic follicles, and no EBV-positive cells were identified in the non-neoplastic area, strongly suggesting an association between FL and the EBV infection.
Similar to a case reported by Gru et al. [2], an acute EBV infection can masquerade as FL in situ. Although some of the follicles were $\mathrm{Bcl}-2$ and $\mathrm{CD} 10$ positive in their case, the microscopic features of lymphomatous transformation were not definite, and EBV was only detected in a few scattered cells in their case. Considering the histological findings and additional studies of the present case (high grade GCs with marked EBER expression in neoplastic GCs and no expression in normal GCs), the association between FL and EBV was more significant and excludes the possibility of an acute EBV infection.

Early phase EBV-positive DLBCL could also have been considered as a diagnosis in our case, which was described by de la Hera Magallanes et al. [3] EBV-positive lymphoproliferative disorders 
are a broad spectrum of diseases from EBV-associated reactive lymphoid hyperplasia to EBV-positive DLBCL, and these B-cell neoplasms can also show various morphological microscopic findings from Hodgkin-like features to monomorphic large B-cell lymphoma-like features. However, our case showed only a follicular growth pattern in the overall excised lymph nodes, and diffuse growth of neoplastic B-cells was not clearly identified. Therefore, the possibility of DLBCL with FL was excluded.

To conclude, rare cases of FL associated with EBV have been reported. Awareness of EBV infection in a patient with FL could contribute to early detection and treatment of this possibly life-threatening disorder. We report a rare case of EBV-positive FL of lymph nodes, hoping that more accumulated cases will support further evaluation for an association between EBV and FL.

\section{REFERENCES}

1. Roschewski M, Wilson WH. EBV-associated lymphomas in adults. Best Pract Res Clin Haematol 2012;25:75-89.

2. Gru AA, Kreisel F, Duncavage E, Nguyen TT, Hassan A, Frater JL. Acute EBV infection masquerading as "In-situ Follicular Lymphoma": a pitfall in the differential diagnosis of this entity. Diagn Pathol 2013;8:100.

3. De la Hera Magallanes AI, Montes-Moreno S, Hernandez SG, HernandezLeon CN, Lopez M, Pajares R, et al. Early phase of Epstein-Barr virus
(EBV)-positive diffuse large B cell lymphoma of the elderly mimicking EBV-positive reactive follicular hyperplasia. Histopathology 2011;59:571-5.

4. Ishtiaq S, Hassan U, Mushtaq S, Akhtar N. Determination of frequency of epstein-barr virus in non- Hodgkin lymphomas Using EBV latent membrane protein 1 (EBV-LMP1) immunohistochemical staining. Asian Pac J Cancer Prev 2013;14:3963-7.

5. Koreishi AF, Saenz AJ, Arcila ME, Hedvat C, Fleming S, Teruya-Feldstein J. Synchronous follicular lymphoma, kaposi sarcoma, and castleman's disease in a HIV-negative patient with EBV and HHV-8 coinfection. Int J Surg Pathol 2011;19:685-91.

6. Seite P, Hillion J, d’Agay MF, Gaulard P, Cazals D, Badoux F, et al. BCL2 gene activation and protein expression in follicular lymphoma: a report on 64 cases. Leukemia 1993;7:410-7.

7. Maeda E, Akahane M, Kiryu S, Kato N, Yoshikawa T, Hayashi N, et al. Spectrum of Epstein-Barr virus-related diseases: a pictorial review. Jpn J Radiol 2009;27:4-19.

8. Sasikala PS, Nirmala K, Sundersingh S, Mahji U, Rajkumar T. Frequency and distribution of Epstein-Barr virus infection and its association with P53 expression in a series of primary nodal non-Hodgkin lymphoma patients from South India. Int J Lab Hematol 2010;32(1 Pt 2):56-64.

9. Masir N, Jones M, Abdul-Rahman F, Florence CS, Mason DY. Variation in BCL2 protein expression in follicular lymphomas without $t(14 ; 18)$ chromosomal translocations. Pathology 2012;44:228-33.

10. Karube K, Guo Y, Suzumiya J, Sugita Y, Nomura Y, Yamamoto K, et al. CD10-MUM1+ follicular lymphoma lacks BCL2 gene translocation and shows characteristic biologic and clinical features. Blood 2007;109:3076-9.

11. Roughan JE, Torgbor C, Thorley-Lawson DA. Germinal center B cells latently infected with Epstein-Barr virus proliferate extensively but do not increase in number. J Virol 2010;84:1158-68. 